\title{
Analytical solution and process analysis of energy pile's heat transfer rate
}

\author{
Jun Yang ${ }^{1}$, Zhenguo Yan ${ }^{1, *}$, Zhengwei Zhang ${ }^{2, *}$, and Shu Zeng ${ }^{1}$ \\ ${ }^{1}$ Tsinghua University, Department of Civil Engineering, 100084 Beijing, China \\ ${ }^{2}$ Zhejiang A\&F University, College of Landscape Architecture, 311300 Hangzhou, China
}

\begin{abstract}
With the ever-increasing energy demand and implications of climate change, the use of energy piles to absorb shallow geothermal energy to regulate room temperature of buildings is considered the best sustainable energy technology, especially in China, and the use of this technology is becoming increasingly popular. At present, studies generally uses the temperature field to analyze the heat transfer performance of the energy pile, which cannot represent the heat transfer rate distribution intuitively. In this study, we used mathematical models to provide an analytical solution to determine the heat transfer rate distribution between the energy pile and surrounding soil. Analysis of the heat transfer process of concrete piles in clay showed that the difference in thermal properties between the energy pile and the surrounding soil affected the whole heat transfer process, especially in the initial stage. The time required to reach the quasi-steady state mainly depended on the pile's volume heat capacity, the thermal diffusivity of the pile and the surrounding soil. In engineering practice, to enhance the heat transfer performance of energy piles, the following measures can be taken: reduce the difference in thermal properties between the energy pile and surrounding soil and increase the distance between energy piles to improve the heat transfer conditions.
\end{abstract}

\section{Introduction}

The issue of climate change is one of the biggest challenges facing the world and is also the key for mankind to achieve sustainable development. The main cause of climate change is the carbon footprint, and buildings, especially high-rise and super-high-rise commercial and residential buildings, account for a large proportion of greenhouse gas (GHG) emissions created by heating, ventilation and air conditioning (HVAC).

To reduce GHG emissions, since the 1980s, the ground source heat pump system (GSHPS) has been used to gradually heat and cool buildings by using shallow geothermal energy, which is effective and conserves energy. Ground heat exchangers (GHEs) of a system embedded in the pile foundation are called energy piles or thermal piles $[1,2]$. Compared with GHEs, the energy pile not only saves the extra drilling cost of borehole GHEs but also reduces the amount of land needed by the horizontal GHEs. The energy pile also is resistant to atmospheric temperature effects and has been increasingly adopted by countries all over the world [3]. The E-terminal of Zurich Airport was opened in September 2003 with energy piles, which are responsible for $85 \%$ of the indoor temperature control requirements and save $25 \%$ of energy consumption compared with the air source temperature control system. After eight years of operation, the investment cost of the energy pile system was recovered [4].

With the use of energy piles, related studies, such as studies of heat transfer performance, mechanical properties and deformations of surrounding soil, have also begun. Heat transfer performance is the major factor that determines the success of the system. Leveraging developments in solid heat conduction, borehole GHES, and computers, many numerical and mathematical models have been developed. Digital twinning can accurately describe the entire heat transfer process of energy piles in soil, but the modeling and the calculation times are long. Meanwhile, the latter simplifies the heat transfer of energy piles, and the solution process is complex but simple to perform. The analytical formula can reflect the influence of various thermal parameters on heat transfer and is suitable for the design and detection of energy piles. The infinite line source model (ILSM) describing borehole GHEs can be used to describe the long-term heat transfer of energy piles [5]. However, compared with borehole GHEs, the diameter of the energy pile is larger, and its influence on heat transfer cannot be ignored [6]. Moreover, there are many pipe configurations, such as U-shaped pipes, W-shaped pipes, and spiral-shaped pipes, each with its own mathematical model and analytical solution [7]. When the pile diameter, inlet water temperature, and flow

*Corresponding authors: yzg17@mails.tsinghua.edu.cn, zwzhang10@126.com 
velocity are the same, the spiral-shaped heat transfer rate is the highest, which is $2.77,2.27$ times higher than that of the other two configurations. The spiral shape is the best pipe configuration for the energy pile [8]. In practice, buried tubes are tied or wound on longitudinal reinforcements [9]. GHEs essentially interact with the surrounding soil at the pile wall. Based on this approximation, many models have been developed, including the solid cylindrical model [10]; line source model in a composite medium [11]; cylindrical model, which considers the difference of thermal properties between the pile and surrounding soil [12]; and so on. In particular, the cylindrical model can fully describe the whole heat transfer process of the pile and surrounding soil via the temperature field and indicates that the increase of the pile's excess temperature, lowering its heat consumption. Under this condition, it is better for the system to transfer heat to the surrounding soil when the heat transfer rate of the energy pile is constant. However, the heat transfer rate is not directly described.

At present, research on the heat transfer rate distribution of energy piles is mainly focused on numerical models rather than mathematical models. In this paper, based on the model from reference [12], the analytical solution of heat transfer rates to both sides of the heat source is proposed, and the heat transfer process of the energy pile is analyzed according to the energy distribution. The remainder of the paper is organized as follows. First, analytical solutions for the heat transfer rates of both sides of the heat source are obtained. Then, from engineering practice, the influence of the energy pile and surrounding soil on heat transfer in the process of heat transfer is analyzed. Finally, according to the conclusions, relevant measures for better heat transfer are proposed.

\section{Analytical solution}

The schematic explaining simultaneous heat transfer from the energy pile wall to both sides is shown in 0 . With the understanding that the thermal properties of the energy pile are different from those of the surrounding soil, the mathematical model of heat transfer and its analytical solution was proposed in reference [12], as shown in Eq. (1) and (2), respectively. At the same time, according to the energy conservation theorem and the heat transfer equation, the heat transfer rates from the heat source to the energy pile and surrounding soil can be expressed as Eq. (3), (4), and (5). By solving the above formulas, the rates of heat transfer from the heat source to the pile and soil at time $t$ can be obtained as Eq. (6) and (7), respectively.

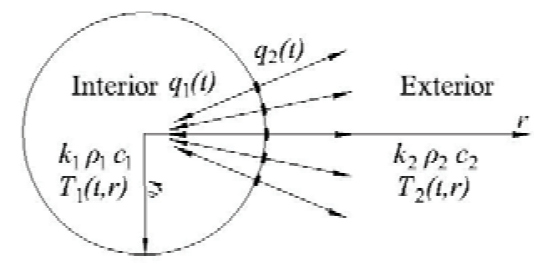

Fig.1 Heat transfer model [12]

$$
\begin{aligned}
& \theta_{1}(r, t)=\frac{q_{l}}{2 \pi^{2} r_{b}} \int_{0}^{\infty}\left(1-e^{-\lambda t}\right) \\
& \times \frac{\left\{J_{0}\left(r \sqrt{\frac{\lambda}{a_{1}}}\right)\left[R(\lambda) J_{0}\left(r \sqrt{\frac{\lambda}{a_{2}}}\right)-H(\lambda) Y_{0}\left(r \sqrt{\frac{\lambda}{a_{2}}}\right)\right]\right\}}{\lambda \sqrt{\lambda}\left[H(\lambda)^{2}+R(\lambda)^{2}\right]} d \lambda \\
& \theta_{2}(t, r)=\frac{q_{l}}{2 \pi^{2} r_{b}} \int_{0}^{\infty}\left(1-e^{-\lambda t}\right) \\
& \times \frac{\left\{J_{0}\left(r_{b} \sqrt{\frac{\lambda}{a_{1}}}\right)\left[R(\lambda) J_{0}\left(r \sqrt{\frac{\lambda}{a_{1}}}\right)-H(\lambda) Y_{0}\left(r \sqrt{\frac{\lambda}{a_{2}}}\right)\right]\right\}}{\lambda \sqrt{\lambda}\left[H(\lambda)^{2}+R(\lambda)^{2}\right]} d \lambda
\end{aligned}
$$

where $J_{n}()$ are Bessel functions of the first order $n, Y_{n}()$ are Bessel functions of the second order $n(n=1,2)$;

$$
\begin{aligned}
& H(\lambda)=\frac{k_{1}}{\sqrt{a_{1}}} J_{1}\left(r_{b} \sqrt{\frac{\lambda}{a_{1}}}\right) J_{0}\left(r_{b} \sqrt{\frac{\lambda}{a_{2}}}\right)-\frac{k_{2}}{\sqrt{a_{2}}} J_{0}\left(r_{b} \sqrt{\frac{\lambda}{a_{1}}}\right) J_{1}\left(r_{b} \sqrt{\frac{\lambda}{a_{2}}}\right) \\
& R(\lambda)=\frac{k_{1}}{\sqrt{a_{1}}} J_{1}\left(r_{b} \sqrt{\frac{\lambda}{a_{1}}}\right) Y_{0}\left(r_{b} \sqrt{\frac{\lambda}{a_{2}}}\right)-\frac{k_{2}}{\sqrt{a_{2}}} J_{0}\left(r_{b} \sqrt{\frac{\lambda}{a_{1}}}\right) Y_{1}\left(r_{b} \sqrt{\frac{\lambda}{a_{2}}}\right) . \\
& \left.2 \pi r_{b} k_{1} \frac{\partial \theta_{1}(t, r)}{\partial r}\right|_{r=r_{b}}=q_{1}(t) \\
& q_{1}(t)=\frac{q_{l}}{\pi} \int_{0}^{\infty} \frac{q_{l}=q_{1}(t)+q_{2}(t)}{\sqrt{a_{1}} \lambda\left[H(\lambda)^{2}+R(\lambda)\right.} k_{r=r_{b}}=q_{2}(t) \\
& \times\left\{\theta_{1}(t, r)\right. \\
& \left.\quad\left(r_{b} \sqrt{\frac{\lambda}{a_{1}}}\right)\left[H(\lambda) Y_{0}\left(r_{b} \sqrt{\frac{\lambda}{a_{2}}}\right)-R(\lambda) J_{0}\left(r_{b} \sqrt{\frac{\lambda}{a_{2}}}\right)\right]\right\} d \lambda
\end{aligned}
$$

$$
q_{2}(t)=q_{l}-q_{1}(t)
$$

In the formula presented above, $\theta_{i}$ is the excess temperature in ${ }^{\circ} \mathrm{C} ; q_{i}$ is the heat transfer rate per length of the material in $\mathrm{kg} / \mathrm{m}^{3} ; \rho_{i}$ is the material density in $\mathrm{kg} / \mathrm{m}^{3} ; c_{i}$ is the specific heat capacity in $\mathrm{J} /\left(\mathrm{kg}^{\circ} \mathrm{C}\right) ; k_{i}$ is the thermal conductivity in $\mathrm{w} /\left(\mathrm{m} \cdot{ }^{\circ} \mathrm{C}\right) ; a_{i}$ is the thermal diffusivity in $\mathrm{m}^{2} / \mathrm{s}$, which is equal to $k_{i} /\left(\rho_{i} c_{i}\right)$; and $i=1$, 2 represent the energy pile and the surrounding soil, respectively. $q_{l}$ is the heating rate per length of the pile in $\mathrm{W} / \mathrm{m}, r$ is the radius of the calculation position in $\mathrm{m}$, $r_{b}$ is the radius of the energy pile in $\mathrm{m}$, and $t$ is the calculation time in $\mathrm{s}$.

At the same time, according to the equations and conditions in reference [12], the analytical solution of the rates in the Cartesian coordinate system is obtained via the Laplace transform and inverse transformation, and the result is also equal to that from Eq. (6), which proves the correctness of the analytical solution.

\section{Impact and analysis}

From Eq. (6), the heat transfer rate to the energy pile itself is affected by the thermal properties of the pile 
and surrounding soil, which are also related to the radius of the pile. Using the analytical formula proposed in this paper, the actual thermal parameters of the energy pile and the soil were selected to study the above correlations in the heat transfer process. Quartz sand concrete were selected as the energy pile material, and clay was chosen as the soil surrounding the pile. Compared with sand, the thermal parameters of clay change with increasing water content. By analyzing dry and saturated clay, the heat transfer of clay under different saturation conditions can be obtained by interpolation [13-15]. The parameters of concrete, saturated clay, and dry clay are shown in Table $1[16$, 17]. For different heat transfer media, the optimum heat transfer rate is different, usually $5-30 \mathrm{~W} / \mathrm{m}$ [18]; we chose a value of $10 \mathrm{~W} / \mathrm{m}$ for this study. The diameter of the pile been defined as $1 \mathrm{~m}$ [19].

Table 1. Thermal parameters of different materials

\begin{tabular}{|c|c|c|c|c|}
\hline Material & $\begin{array}{c}k \\
\mathrm{Wm}^{-1} \mathrm{~K}^{-1}\end{array}$ & $\begin{array}{c}c \\
\mathrm{Jkg}^{-1} \mathrm{~K}^{-1}\end{array}$ & $\begin{array}{c}\rho \\
\mathrm{kgm}^{-3}\end{array}$ & $\begin{array}{c}a \\
\mathrm{~m}^{2} \mathrm{~s}^{-1}\end{array}$ \\
\hline Concrete & 2 & 1000 & 2500 & $\begin{array}{c}8 \\
\times 10^{-7}\end{array}$ \\
\hline $\begin{array}{c}\text { Saturated } \\
\text { clay }\end{array}$ & 1.70 & 1325 & 2000 & $\begin{array}{c}6.42 \\
\times 10^{-7}\end{array}$ \\
\hline Dry clay & 0.50 & 270 & 1680 & $\begin{array}{c}1.10 \\
\times 10^{-6}\end{array}$ \\
\hline
\end{tabular}

The ratio of the heat transfer rate to the surrounding soil at moment $t$ is defined as $R_{o}$, as shown in Eq. (8).

$$
R_{o}(t)=q_{2}(t) / q_{l}(t)
$$

When the energy pile transfers heat in dry clay and saturated clay, the change in $R_{o}$ with time is shown in 0 , and $R_{o}$ is expressed as $R_{o d}$ and $R_{o s}$, respectively. When the heat transfer period is 1 hour, the value of $R_{o d}$ is less than $1 / 4$, while the value is close to 0.5 ; the latter is almost twice as much as the former. When the value of $R_{o}$ reaches 0.5 , the former needs 84 hours, while the latter only takes 4 hours. During a heat transfer period of three months, the value of $R_{o d}$ reaches $0.6,0.7,0.8$ and 0.9 requires 144 hours, 239 hours, 403 hours, and 752 hours, respectively. With increasing heat transfer time, the value of $R_{o d}$ increases, but the growth rate slows. Finally, the value approaches to 1 but is less than 1 . The value of $R_{o s}$ is higher than that of $R_{o d}$ until the heat transfer time reaches 40 days and takes 23 hours, 54 hours, 118 hours, and 408 hours to reach $0.6,0.7$, 0.8 , and 0.9 , respectively. During this period, the growth rate gradually decreases. On the $40^{\text {th }}$ day, the two values are the same, equal to 0.93 . Later, the value of $R_{o s}$ is less than that of $R_{o d}$, approximately 0.95 , when the heat transfer time is 3 months.

From 0, it can also can be seen that in the initial stage of heat transfer, compared with the values of $R_{o d}$ and $R_{o s}$, the closer the thermal properties of concrete and clay, the greater the value of $R_{o}$. When the thermal properties of dry clay are selected for both the energy pile and the soil outside, the value of $R_{o}$ is greater than that of $R_{o s}$ at the same heat transfer time.

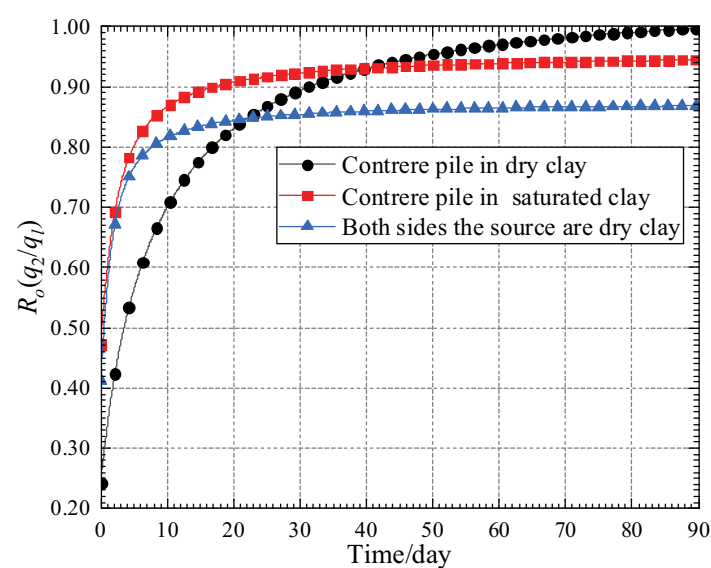

Fig.2 The ratio of the heat transfer rate $R_{o}$

As the heat transfer continues, the value of $R_{o s}$ smaller than that of $R_{o d}$ due to the thermal property differences between the concrete and the clay with varying moisture. Dry clay, with a larger thermal diffusivity and smaller specific heat capacity, has a larger heat transfer range and higher excess temperature, as shown in 0 . Based on the model assumption of the single pile, the pile's cross-section is limited and the surrounding soil is infinity, so the heat needed by the soil increases with the heat transfer time. Meanwhile, when the heat transfers in saturated clay, the heat required to expand the heat transfer range increases more slowly than in dry clay. During the three-month heat transfer period, the value of $R_{o s}$ approaches $95 \%$.

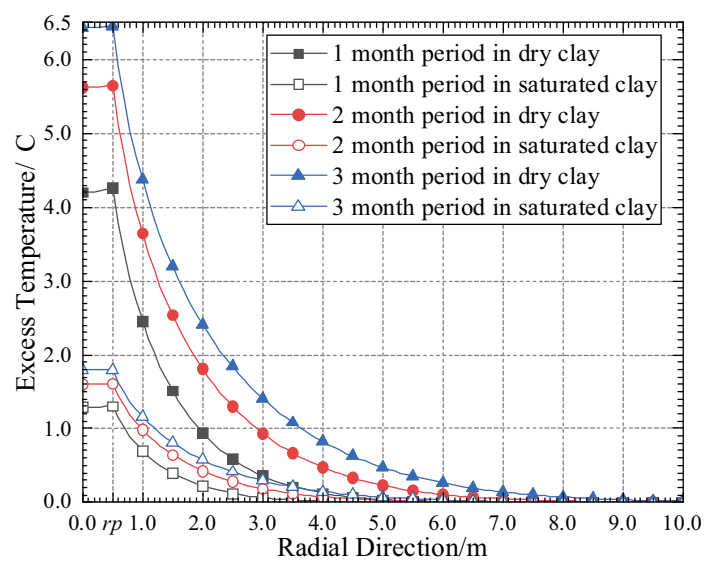

Fig.3 Excess temperature distribution

In practical engineering applications, energy piles often appear in the form of pile groups. According to the superposition principle, the effective heat transfer ratio is $1 / 2$ of the distance between piles, which contrasts with the model assumption of infinity outside the pile. Therefore, the value of $R_{o d}$ tends to be 1 , as shown in 0 but cannot be reached. Furthermore, because of the heat accumulation in the energy pile and surrounding soil, whose excess temperature rose, the temperature difference smaller between the inlet and outlet, making the heat transfer rate smaller under the 
condition of a constant flow velocity and inlet temperature. In comparison, saturated clay is more suitable for the heat transfer of energy piles. When using the energy pile, the saturation of soil can even be increased by an artificial intervention to ensure the heat dissipation capacity of the energy piles [20]. The other way to enhance the heat transfer performance is using an alternate arrangement of the energy pile and common pile foundation during the design process to increase the distance between the energy piles.

Moreover, when performing the thermal response test, $t_{c}=5 r_{p}^{2} / a_{2}$ is often used as the time for the energy pile to reach the quasi-steady state [21]. For saturated clay and dry clay, the corresponding times are 541 hours and 343 hours, respectively. The time needed for saturated clay obtained by this equation is less than the time needed for dry clay. However, according to Fig. 1 , during the abovementioned time, the values of $R_{o}$ are $91.3 \%$ and $81.3 \%$. For saturated clay, the value close to $95 \%$ almost reached the steady state; for dry clay, at that time, there is still $20 \%$ of heat consumption in the pile, far from reaching the quasi-steady state. These conclusions are illustrated in 0 . It can be concluded that if there is a great difference in thermal properties between the energy pile and the soil, then ignoring the difference is not appropriate when calculating the quasi-steady state time, and the difference should be fully considered to define the quasi-steady state of the energy pile.

\section{Conclusion}

Based on the law of conservation of energy, this paper presents a mathematical model and its analytical solution for the heat transfer rate distribution and analyzes the heat transfer process of concrete energy piles in clay. Through comparative analysis, the conclusions are as follows:

1. During the whole heat transfer period, the energy pile's heat consumption cannot be ignored. At the initial stage, the heat transfer to the pile is greater than that transferred to the surrounding soil and increases with the increasing difference in thermal parameters between the pile and surrounding soil. With increasing heat transfer time, the heat transfer rate to the surrounding soil increases gradually.

2. The heat transfer performance of clay varies with its saturation. In the initial stage, the heat transfer rate to clay increases with increasing clay saturation, while in the later stage of heat transfer, the heat transfer to clay decreases with increasing clay saturation. At the same time, as the clay saturation decreases, the excess temperatures of the pile and soil increase, and the range of the temperature field increases.

3. As a pile foundation, the energy pile always appears as a group of piles, and the heat transfer radius is limited. When the clay saturation is small, it may cause temperature accumulation of the energy pile and its surroundings and weaken the heat transfer capacity of the energy pile. In practical engineering, taking measures to increase the saturation of clay is an effective way to increase the heat transfer capacity of energy piles. Alternatively, during the design process, an alternate arrangement of energy piles and common pile foundations can enhance the heat transfer performance by increasing the distance between energy piles.

4. At present, the conventional formula for calculating the quasi-steady time of the energy pile ignores the influence of the difference in the thermal properties between the energy pile and the surrounding soil. When the difference is large, the calculation result of the formula is quite different from reality. The formula proposed in this paper is more accurate for describing the state of an energy pile in the process of heat transfer.

\section{References}

[1] H. Brandl, Geotechniqueb 56(2), 81-122 (2006)

[2] F. Loveridge, W. Powrie, Geotech. Eng. 166(2), 178-196 (2013)

[3] M. de Moel, P.M. Bach, A. Bouazza, R.M. Singh, J.O. Sun, Renew. Sust. Energ. Rev. 14(9), 2683$2696(2010)$

[4] L. Laloui, A. Di Donna, Proc. Inst. Civil Eng.-Civil Eng. 164(4), 184-191 (2011)

[5] H. Carslaw, J. Jaeger, Heat conduction in solids (Oxford Univ. Press, England, 1947)

[6] P. Bourne-Webb, European Geothermal Congress (Pisa, Italy, 2013)

[7] G.Q. Huang, X.F. Yang, Y.J. Liu, C.L. Zhuang, H.Y. Zhang, J. Lu, Energy Build. 158, 1241-1256 (2018)

[8] B. Bezyan, S. Porkhial, A.A. Mehrizi, Appl Therm Eng. 87, 655-668 (2015)

[9] S. Park, C. Sung, K. Jung, B. Sohn, A. Chauchois, H. Choi, Appl Therm Eng. 90, 1061-1071 (2015)

[10] Y. Man, H.X. Yang, N.R. Diao, J.H. Liu, Z.H. Fang, Int. J. Heat Mass Transf. 53(13-14), 2593-2601 (2010)

[11] M. Li, A.C.K. Lai, Energ. 38(1), 255-263 (2012)

[12] J. Yang, Z. Yan, X. Li, Z. Zhang, S. Zeng, Int. J. Heat Mass Transf. 152, 119559 (2020)

[13] N.H. Abu-Hamdeh, Biosyst. Eng. 86(1), 97-102 (2003)

[14] X. Li, Y. Chen, Z. Chen, J. Zhao, , Energy Build. 38(5), 543-547 (2006)

[15] Z. Liu, J. Xu, X. Li, J. Wang, Mechanisms of biochar effects on thermal properties of red soil in south China, Geoderma 323, 41-51 (2018)

[16] MOHURD, Code for design of contrete structures (China Architecture \& Building Press, Beijing, 2010)

[17] GSHPA, Thermal pile design, installation and materials standards (Ground Source Heat Pump Association Milton Keynes, UK, 2012)

[18] I. Staffell, D. Brett, N. Brandon, A. Hawkes, Energy Environ. Sci. 5(11), 9291-10007 (2012)

[19] Q. Zhao, B.M. Chen, M.C. Tian, F. Liu, Energ. 162, 787-797 (2018) 
[20] J. Zhao, Y. Li, J. Wang, Energy Procedia 104, 413 418 (2016)

[21] MHURDPR Technical standard for utilization of geothermal energy through piles (China Architecture \& Building Press, Beijing, 2018) 